\title{
HIGHLY SENSITIVE MICROFLUIDIC SENSOR ARCHITECTURE FOR SERS MEASUREMENTS OF HORMONES
}

\author{
${ }^{1}$ Vasilii BURTSEV, ${ }^{1,2}$ Elena MILIUTINA, 1,2Mariia ERZINA, ${ }^{1}$ Vaclav ŠVORČíK, \\ 1,2Oleksiy LYUTAKOV \\ ${ }^{1}$ VŠCHT - University of Chemistry and Technology, Prague, Czech Republic, EU, lyutakoo@vscht.cz \\ ${ }^{2}$ Tomsk Polytechnic University, Research School of Chemistry and Applied Biomedical Sciences, Tomsk,
} Russia

https://doi.org/10.37904/nanocon.2020.3743

\begin{abstract}
Today, there is considerable interest in the development of microfluidic chips that can be integrated into various analytical devices and further used for chemical or biological research. The microfluidic approach can reduce the size of all types of analytical laboratory equipment's and improve their technological performances. In addition, in combination with Surface Enhanced Raman Scattering (SERS), microfluidic devices can detect unprecedentedly low concentrations of targeted analytes. SERS provides a significant amplification of the nonlinear optical response of a molecule adsorbed on a suitable plasmon-active (most often silver or gold) surface (for example nanograting, nanoparticles, etc.). On the other hand, microfluidics allows a substantial reduction in the volume of sample required for analysis. In this work, an approach based on a combination of SERS and microfluidics was implemented and enables the detection of low concentrations of the target analyte - hormones. Proposed preparation of microfluidic chip comprises several subsequent steps: (i) preparation of microfluidic platform using 3D printing; (ii) laser assisted creation of patterned surface, further covered by a thin Au layer, ensuring the excitation of surface plasmon polariton in the microfluidic canal, (iii) subsequent grafting of $-\mathrm{NH}_{2}$ groups, and their activation for hormones entrapping. The rapid, reproducible, and ultrasensitive detection of hormones - Bisphenol, Estradiol, Estriol $\left(<10^{-6} \mathrm{M}\right)$ was achieved.
\end{abstract}

Keywords: Lab-on-a-chip, SERS, 3D printing, microfluidics system, hormones

\section{INTRODUCTION}

Sensors that determine the concentration of hormones, especially estrogens, are of particular interest for medical and environmental research [1]. Even a small, uncontrolled use of hormones by a person can cause adverse health effects, since certain types of hormones have been shown to cause cancer, heart disease and stroke [2]. Thus, there is a need for the determination of hormones to control their concentration in water and other biological fluids $[3,4]$.

Today, there are several approaches to detecting hormones such as liquid chromatography and mass spectrometry $[5,6]$. However, they are quite complex, expensive, and time-consuming to analyse and results interpretation. Also, in recent years, sensors operating on the principle of surface plasmon resonance have become popular [7], but they have low selectivity and detection limit, which restricts their practical utilization. Therefore, the development of a plasmon-based (bio)sensor that will make it possible to carry out analysis quickly and reliably even with a small sample volume remains unsolved task.

The microfluidic approach makes it possible to reduce the size of all types of analytical laboratory equipment and improve their technological properties $[8,9]$. In addition, when combined with surface enhanced Raman scattering (SERS), microfluidic devices can detect unprecedented low concentrations of target analytes even with a small sample volume [10-13]. SERS provides a significant enhancement of the nonlinear optical response of a molecule adsorbed on a suitable metal (most often silver or gold) surface, while microfluidics can significantly reduce the sample requirements for analysis. In this work, we propose an approach based on 
a combination of SERS and microfluidics, which made it possible to identify low concentrations of the target analytes - hormones.

\section{EXPERIMENTAL}

\subsection{Materials}

The 3D printable polymer, Clear resin, was purchased from Formlabs, USA. The solution of photoresist, Su-8, was obtained from Microchem, Germany, Isopropyl alcohol ( $\geq 98.5 \%)$, deionized water, tert-Butyl nitrite $(90 \%)$, methanol (reagent grade, $\geq 99,8 \%$ ), p-Toluenesulfonic acid monohydrate (ACS reagent, $\geq 98.5 \%$ ), Bisphenol $A(\geq 99 \%), \beta$-Estradiol ( $\geq 98 \%)$ and Estriol $(\geq 97 \%)$ were purchased from Sigma-Aldrich and used without further purification. The Au target (purity $4 \mathrm{~N}$ ) was purchased from Safina. 4-aminobenzenediazonium tosylate (ADT$\mathrm{NH}_{2}$ ) was prepared according to published procedure [14].

\subsection{Samples preparation}

\section{Microfluidic chip preparation}

The microfluidic chip was made using $3 \mathrm{D}$ printing. The model for printing was designed in the program COMSOL Multiphysics. After fabrication, the microfluidic micromixer was washed with isopropyl, dried at $40^{\circ}$ $\mathrm{C}$ for $24 \mathrm{~h}$ and additionally irradiated by UV-source for $30 \mathrm{~min}$.

Su-8 film $(10 \mu \mathrm{m})$ were spin-coated on the surfaced of microfluidic chip (1000 rpm, $10 \mathrm{~min})$, dried at $60{ }^{\circ} \mathrm{C}$ for $24 \mathrm{~h}$, irradiated by UV-source for $30 \mathrm{~min}$, and annealed at $90{ }^{\circ} \mathrm{C}$ for $2 \mathrm{~h}$. Pattering of Su-8 surface was performed using linearly polarized excimer laser beam from COMPexPro 50F KrF (emission wavelength 248 $\mathrm{nm}$, laser fluence $10 \mathrm{~mJ} \times \mathrm{cm}^{-2}, 3500$ total number of pulses, beam incident angle $40^{\circ}$ ).

The Au thin films were deposited onto the patterned surface by vacuum sputtering (thickness $30 \mathrm{~nm}$ ).

\section{Diazonium modification and diazotization}

Au grating was electrochemically modified in $3 \mathrm{mM}$ fresh water solutions of ADT-NH 2 without addition of any electrolytes, under the potential $-5 \mathrm{~V}$ for $6 \mathrm{~min}$. After modification, chips were rinsed under sonication sequentially with deionized water, ethanol, and acetone for $15 \mathrm{~min}$, and dried in desiccator for $3 \mathrm{~h}$.

The sample was immersed in a $15 \mathrm{ml}$ methanol solution of $\mathrm{p}$-Toluenesulfonic acid monohydrate and $0.3 \mathrm{ml}$ of tert-Butyl nitrite was added. After activation chips were rinsed under sonication with $\mathrm{MeOH}$ for 15 min and dried in desiccator for $3 \mathrm{~h}$.

\subsection{Measurement Techniques}

\section{Raman Spectroscopy Investigations-Concentration Dependence}

For microfluidic SERS measurements, the chips were pumped with water solutions of Bisphenol, Estradiol and Estriol. Raman spectra were collected on portable ProRaman-L spectrometer (Laser power $35 \mathrm{~mW}$ ) with 785 $\mathrm{nm}$ excitation wavelengths. Spectra were measured in the $2000-400 \mathrm{~cm}^{-1}$ wavenumber range with $60 \mathrm{~s}$ accumulation time and averaged 3 times.

\section{RESULTS AND DISCUSSION}

The preparation of the proposed SERS microfluidic chips consists of several stages, as schematically shown in Figure 1. First, a 3D microchannel was made using 3D printing technology and covered with a thin layer of Su-8. In the second stage, the periodic structure was created on the Su-8 by irradiation with excimer laser and covered with a thin layer of gold. Further the chip surface was grafted with $-\mathrm{NH}_{2}$ groups and activated to obtain a $\mathrm{N}=\mathrm{N}^{+}$reactive sites, that allows hormones capturing. 


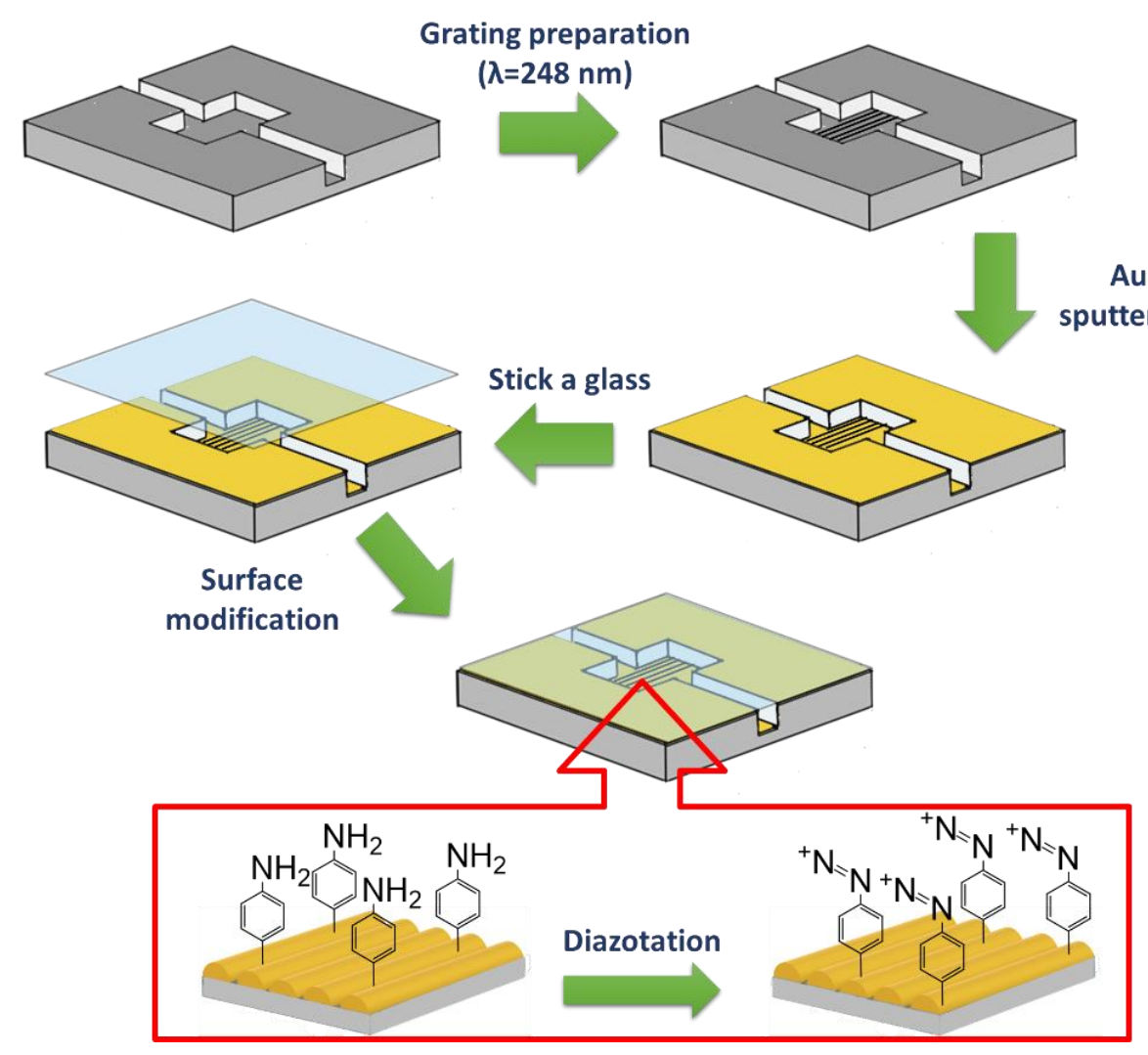

Figure 1 Schematic representation of the preparation of hybrid microfluidic sensor
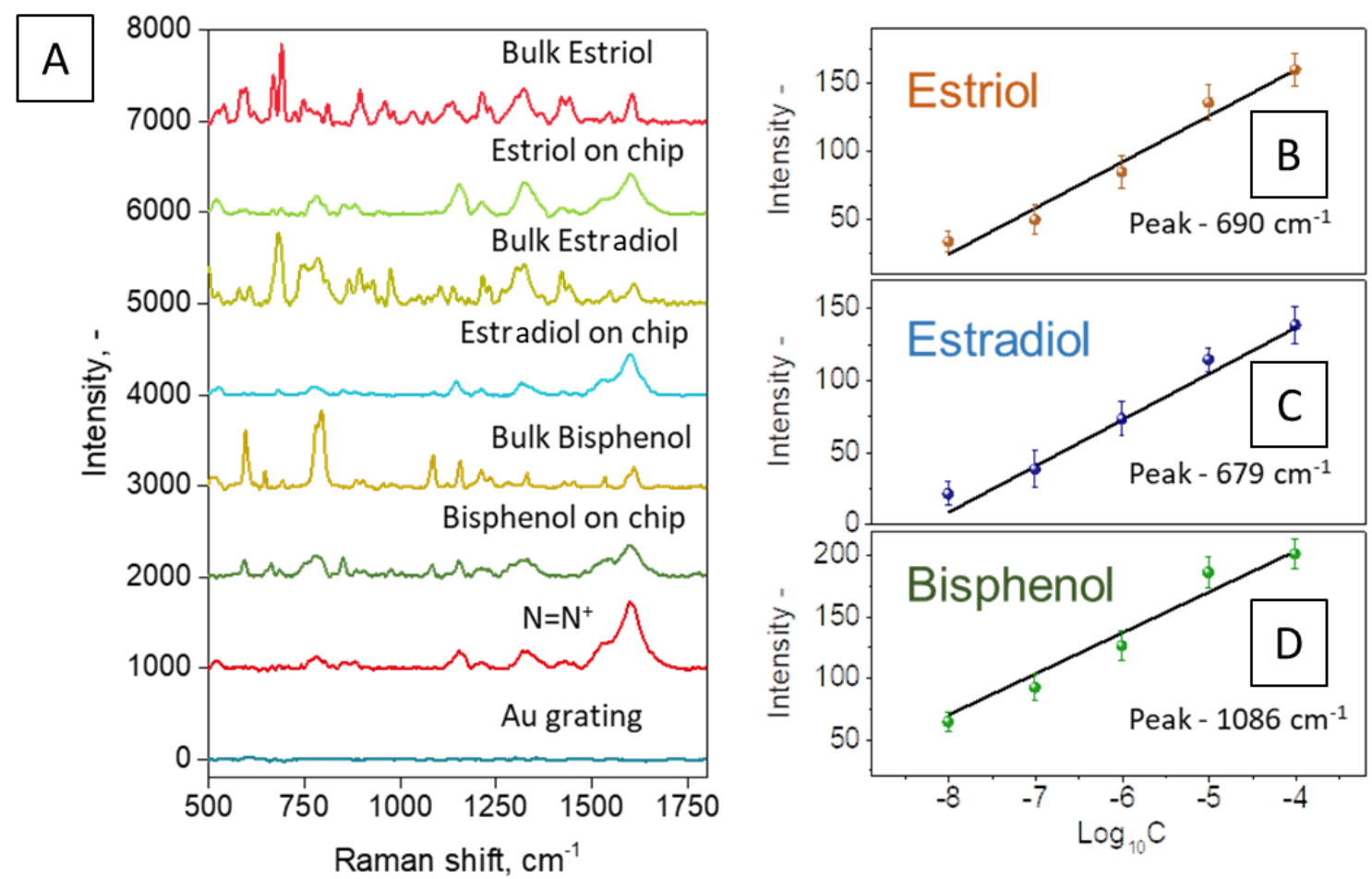

Figure 2 (A) SERS spectra measured on bulk hormones and hormones, entrapped on chip $\left(10^{-6} \mathrm{M}\right)$ in microfluidic regime. (B) dependency of characteristic Estriol peak intensity on it initial concentration in water; (C) dependency of characteristic Estradiol peak intensity on it initial concentration in water; (D) dependency of characteristic Bisphenol peak intensity on it initial concentration in water. 
In the next step, we used two natural steroidal estrogens (estradiol and estriol) and one non-steroidal estrogen (bisphenol A) as model analytes for the study. We compare the SERS spectra, measured on SERS active substrate after hormones capturing in microfluidic regime with Raman spectra of targeted analyte in bulk. In all cases, the perfect coincidence of peak position and relative intensities (after spectra subtraction) was observed, indicating the success of proposed experimental route. In turn, Figures 2B-D show the calibration curves, prepared with more intensive, characteristic hormones SERS band. From calibration curves it is evident, that all used hormones were successfully entrapped detected up to $10^{-8} \mathrm{M}$ concentration. Comparison of obtained measurements uncertainty with typical errors from Figures 2B-D allows us to claim that by the proposed method it is possible to determine investigated hormones concentration with high precision.

\section{CONCLUSION}

In this work, we present the novel design of microfluidic-based chip for SERS detection of Bisphenol A, $\beta$ Estradiol and Estriol. The microfluidic chip was designed in Comsol and fabricated using 3D printing. The proposed detection approach is simple, fast, sensitive, and versatile, with great potential in hormone detection applications to monitor the safety of water and environment.

\section{ACKNOWLEDGEMENTS}

This work was supported by the project Internal Grant Agency 126882007.

\section{REFERENCES}

[1] HAN, X.X., PIENPINIJTHAM, P., ZHAO, B., and OZAKI, Y. Coupling Reaction-Based Ultrasensitive Detection of Phenolic Estrogens Using Surface-Enhanced Resonance Raman Scattering. Analytical Chemistry. 2011, vol. 83, no. 22, pp. 8582-8588.

[2] TAYLOR, A.E., KHOURY, R.H., and CROWLEY, W.F. A comparison of 13 different immunometric assay kits for gonadotropins: implications for clinical investigation. The Journal of Clinical Endocrinology \& Metabolism. 1994, vol. 79, no. 1, pp. 240-247.

[3] GJERGJIZI, B., ÇOĞUN, F., YILDIRIM, E., ERYILMAZ, M., SELBES, Y., SAĞLAM, N., and TAMER, U. SERSbased ultrafast and sensitive detection of luteinizing hormone in human serum using a passive microchip. Sensors and Actuators B: Chemical. 2018, vol. 269, pp. 314-321.

[4] WANG, R., CHON, H., LEE, S., CHENG, Z., HONG, S.H., YOON, Y.H., and CHOO, J. Highly Sensitive Detection of Hormone Estradiol E2 Using Surface-Enhanced Raman Scattering Based Immunoassays for the Clinical Diagnosis of Precocious Puberty. ACS Applied Materials \& Interfaces. 2016, vol. 8, no. 17, pp. 10665-10672.

[5] STOPFORTH, A., BURGER, B.V., CROUCH, A.M., and SANDRA, P. The analysis of estrone and 17ß-estradiol by stir bar sorptive extraction-thermal desorption-gas chromatography/mass spectrometry: Application to urine samples after oral administration of conjugated equine estrogens. Journal of Chromatography B. 2007, vol. 856, no. 1 , pp. $156-164$.

[6] STANCZYK, F.Z., CLARKE, N.J. Advantages and challenges of mass spectrometry assays for steroid hormones. The Journal of Steroid Biochemistry and Molecular Biology. 2010, vol. 121, no. 3, pp. 491-495.

[7] MUDA, N.E., ABU BAKAR, MOHD.A. and MAJLIS, B.Y. The Use of Electronic Sensor in Hormone Analysis. The Malaysian Journal of Medical Sciences: MJMS. 1999, vol. 6, no. 2, pp. 12-16.

[8] CASTILLO-LEÓN, J. Microfluidics and Lab-on-a-Chip Devices: History and Challenges. In: J. CASTILLO-León and W.E. SVENDSEN, eds. Lab-on-a-Chip Devices and Micro-Total Analysis Systems: A Practical Guide. 2015, pp. $1-15$.

[9] JEON, J., CHOI, N., CHEN, H., MOON, J.-I., CHEN, L., and CHOO, J. SERS-based droplet microfluidics for highthroughput gradient analysis. Lab on a Chip. 2019, vol. 19, no. 4, pp. 674-681. 
[10] BURTSEV, V., MILIUTINA, E., ERZINA, M., KALACHYOVA, Y., ELASHNIKOV, R., SVORCIK, V., and LYUTAKOV, O. Advanced Design of Microfluidic Chip Based on SPP-LSP Plasmonic Coupling for SERS Detection with High Sensitivity and Reliability. The Journal of Physical Chemistry C, 2019, vol. 123, no. 50, pp. 30492-30498.

[11] PU, H., XIAO, W., and SUN, D.-W. SERS-microfluidic systems: A potential platform for rapid analysis of food contaminants. Trends in Food Science \& Technology. 2017, vol. 70, pp. 114-126.

[12] MILIUTINA, E., GUSELNIKOVA, O., CHUFISTOVA, S., KOLSKA, Z., ELASHNIKOV, R., BURTSEV, V., POSTNIKOV, P., SVORCIK, V., and LYUTAKOV, O. Fast and All-Optical Hydrogen Sensor Based on GoldCoated Optical Fiber Functionalized with Metal-Organic Framework Layer. ACS Sensors. 2019, vol. 4, no. 12, pp. 3133-3140.

[13] GAO, R., KO, J., CHA, K., HO JEON, J., RHIE, G., CHOI, J., DEMELLO, A.J., and CHOO, J. Fast and sensitive detection of an anthrax biomarker using SERS-based solenoid microfluidic sensor. Biosensors and Bioelectronics, 2015, vol. 72, pp. 230-236.

[14] GUSELNIKOVA, O., POSTNIKOV, P., ELASHNIKOV, R., TRUSOVA, M., KALACHYOVA, Y., LIBANSKY, M., BAREK, J., KOLSKA, Z., ŠVORČíK, V., and LYUTAKOV, O. Surface modification of Au and Ag plasmonic thin films via diazonium chemistry: Evaluation of structure and properties. Colloids and Surfaces A: Physicochemical and Engineering Aspects. 2017, vol. 516, pp. 274-285. 